\title{
Another Assessment of Fat Degeneration of Retracted Supraspinatus Muscle
}

\author{
Yeon-Seok Jeong, Jae-Kwang Yum ${ }^{\varpi}$, Sang-Yoon Park \\ Department of Orthopaedic Surgery, Sanggye Paik Hospital, Inje University School of Medicine, Seoul, Korea
}

Background: The purpose of this study was to assess the relevance of preoperative magnetic resonance imaging (MRI) evaluation by occupation ratio (OR) at maximum diameter of supraspinatus muscle.

Methods: Patients from the Inje University Sanggye Paik Hospital who received rotator cuff repair and underwent pre- and postoperative MRI were selected as subjects of this study. On T1-weighted MRIs, OR of fat and muscle at Y-shaped view, OR at a location on supraspinatus muscle where its diameter was maximum on coronal view, and pre- and postoperative Goutallier Classification and changes in the tangent sign were measured. Statistical significance of postoperative OR was assessed regarding time from symptom onset to surgery, size of rotator cuff tear, preoperative OR, and the difference between ORs measured at maximum diameter of supraspinatus muscle and Yshaped view.

Results: Preoperative OR at Y-shaped view was $52.28 \pm 8.57$ (32.5-65.3). Preoperative OR difference between maximum diameter and Y-shaped view was $13.76 \pm 10.51$ (2.38-42.04), and Pearson correlation coefficient was 0.604 ( $p=0.001)$. Postoperative OR at Y-shaped view was $63.77 \pm 9.35$ (37.3-76.1). Pearson correlation coefficient of pre- and postoperative Goutallier Classification was -0.579 ( $p=0.002)$ and Pearson correlation coefficient of the postoperative difference between ORs measured at maximum diameter of supraspinatus muscle and Y-shaped view was $-0.386(p=0.047)$.

Conclusions: Fatty degeneration of supraspinatus muscle in rotator cuff tear patients should be evaluated not only in the conventional Yshaped view, but also at location of maximum diameter of supraspinatus muscle to establish patients' therapeutic plan.

(Clin Shoulder Elbow 2018;21(4):200-206)

Key Words: Rotator cuff tears; Supraspinatus muscle; Occupation ratio; Retraction; Fat degneration

\section{Introduction}

Magnetic resonance imaging (MRI) is a noninvasive study and is known to be the most useful method to diagnosis the lesions of the shoulder. The condition of shoulder muscles, the degree of retraction or atrophy, and the state of the joint capsule as well as the size of a rotator cuff tear can be identified using MRI. ${ }^{1)}$ Since fatty degeneration and muscle atrophy of rotator cuff muscles are closely related to the assessment of moderate rotator cuff disease, treatment plans, and prognostic factors, they should be carefully and accurately evaluated. ${ }^{2)}$ Goutallier et al. ${ }^{3)}$ provided the classification method of fatty degeneration, and
Gladstone et al. ${ }^{4)}$ showed that fatty degeneration of the rotator cuff is one of the prognostic factors of clinical results of rotator cuff repair. Thomazeau et al. ${ }^{5)}$ proposed a method for evaluation of atrophy of the supraspinatus muscle. In addition, many researchers have shown that preoperative fatty degeneration and atrophy affect the incidence of re-rupture after surgery and surgical outcomes. ${ }^{6,7}$ However, in patients with rotator cuff tear, the supraspinatus muscle is pulled toward the proximal portion especially if the rupture of the supraspinatus muscle is present. In this case, it has been reported that the evaluation results of the degrees of fatty degeneration and atrophy of the supraspinatus muscle by the Y-shaped view are different from those of

Received August 27, 2018. Revised October 5, 2018. Accepted October 9, 2018.

Correspondence to: Jae-Kwang Yum

Department of Orthopaedic Surgery, Inje University Sanggye Paik Hospital, 1342 Dongil-ro, Nowon-gu, Seoul 01757, Korea

Tel: +82-2-950-1027, Fax: +82-2-950-1429, E-mail: Yumccf@hanmail.net, ORCID: https://orcid.org/0000-0002-0286-7981

IRB approval: Inje University Sanggye Paik Hospital (SGPAIK 2018-08-030).

Financial support: None. Conflict of interests: None. 
patients without the rupture of the supraspinatus muscle. ${ }^{8)}$ Vidt et al. ${ }^{9)}$ claimed that three-dimensional evaluation of muscles is required rather than evaluation of fatty degeneration only in the Y-shaped view. In addition, Gerber et al., ${ }^{10)}$ Yamaguchi et al., ${ }^{11)}$ and Chung et al. ${ }^{12)}$ reported that atrophy of the supraspinatus muscle was improved immediately after surgery, but Deniz et al. ${ }^{13)}$ and Liem et al. ${ }^{6}$ reported that there were no changes in muscle atrophy or fatty degeneration before and after surgery. A quantitative analysis of Gerber et al. ${ }^{14)}$ showed that muscle separation did not affect hemoperfusion or change the amount of muscle fibers. These results of previous studies led the authors to question whether the evaluation of the supraspinatus muscle in the Y-shaped view leads to correct interpretations in both the patients with the retracted supraspinatus muscle and those with the nonretracted supraspinatus muscle. In the Inje University Sanggye Paik Hospital, we perform immediate postoperative MRI of patients after rotator cuff repair. The comparison between preoperative MRI evaluations and postoperative MRI outcomes showed that there were changes in the degrees of fatty degeneration and atrophy of the supraspinatus muscle in many patients. It is thought that when preoperative MRI shows that the supraspinatus muscle is pulled toward the proximal portion due to the rupture or chronic rupture, the conventional assessment of fatty degeneration in the Y-shaped view is not sufficient to evaluate the state of the supraspinatus muscle. Therefore, in this study, we aimed to evaluate the need for the evaluation of fatty degeneration not only in the Y-shaped view, where the evaluation region becomes different depending on the degree of muscle pull of the supraspinatus muscle, but also at the maximum diameter location of the supraspinatus muscle to evaluate the overall fatty degeneration of the pulled supraspinatus muscle.

\section{Methods}

\section{Subjects}

Among 82 patients who were diagnosed with rotator cuff tear and underwent rotator cuff repair in our institution from January 2012 to December 2016, 37 patients who did not undergo immediate postoperative MRI and 18 patients in whom fatty degeneration was not detected on MRI were excluded, and a total of 27 patients were included in this study. The state of the muscle was evaluated in comparison with the preoperative state. The mean age of the patients was 56.1 years (median: 58 years; range: 40-67 years). The subjects consisted of 13 women and 14 men, and the right shoulder was involved in 20 cases and the left shoulder in 7 cases. The mean time from symptom onset to surgery was $21.15 \pm 31.84$ months (0.75-120 months). The

Table 1. Demographic

\begin{tabular}{lcc}
\hline \multicolumn{1}{c}{ Variable } & Value & $p$-value \\
\hline Age $(\mathrm{yr})$ & $56.1(58,40-67)$ & 0.609 \\
Orientation & & \\
\hline Rightt & 20 & \\
\hline Left & 7 & \\
\hline Sex & 14 & \\
\hline Male & 13 & $0.009^{*}$ \\
\hline Female & $21.15 \pm 31.84(0.75-120)$ & 0.962 \\
\hline Symptom onset (mo) & $163.37 \pm 7.49(150-182)$ & 0.973 \\
\hline Height $(\mathrm{cm})$ & $65.69 \pm 12.06(48-94)$ & 0.255 \\
\hline Weight $(\mathrm{kg})$ & $4.37(5,3-6)$ & \\
\hline Postoperative MRI day & & \\
\hline
\end{tabular}

Values are presented as mean (median, range), number only, or mean \pm standard deviation (range). Kolmogorov-Smirnov test.

MRI: magnetic resonance imaging.

${ }^{*}$ Statistically significant.
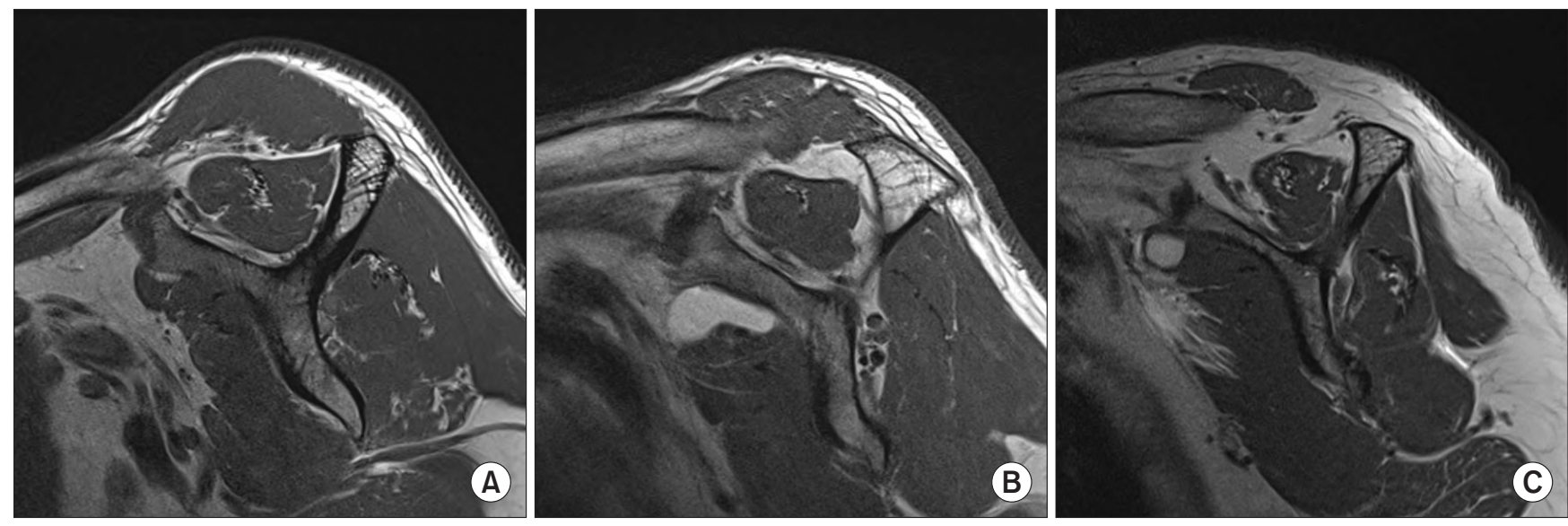

Fig. 1. Magnetic resonance imaging (MRI) of rotator cuff tear patients. Preoperative MRI shows degrees of fat degeneration in supraspinatus muscle; grade I by Goutallier sign (A), grade II by Goutallier sign (B), grade III by Goutallier sign (C). 
mean follow-up period was 4.37 days (median: 5; range: 3-6) (Table 1).

\section{Evaluation Methods}

Radiographic evaluation was performed with 3-mm-thick T1weighted MRIs of the axial, oblique coronal, and oblique sagittal planes (repetition time, 450-750 msec; echo time, 10-12 msec; the field of views, $150 \times 150 ; 448 \times 403$ pixel matrix) using 3.0T MRI (MAGNETOM ${ }^{\circledR}$ Skyra; Siemens Medical, Berlin, Germany). Immediate postoperative MRI was performed one day after removing the hemavoc drain after waiting until less than $10 \mathrm{ml}$ of fluid was drained through the hemovac drain inserted during surgery. The tendon atrophy was assessed by measuring the length from the sulcus of the greater tuberosity to the outer end of the ruptured tendon in the coronal plane. Fatty degeneration of the supraspinatus muscle was also assessed using Goutallier's classification adapted by Fuchs et al. ${ }^{15)}$ on pre- and postoperative MRI. In the Y-shaped view, fatty degeneration of the muscle is classified as grade 0 if fat is not seen, grade I if fatty streaks are observed, grade II if there is some amount of fat but there is less fat than muscle, grade III if there are equal amounts of fat and muscle, and grade IV if there is more fat than muscle (Fig. 1). In addition, the modified tangent sign presented by Liem et al. ${ }^{6}$ was also examined. The modified the tangent sign was categorized as grade I if the supraspinatus muscle was superior to the tangent line, grade II if the muscle touched the line, and grade III if the muscle was below the line, respectively (Fig. 2). The occupation ratio (OR) of the supraspinatus muscle was assessed in the Y-shaped view, where the scapula body and the spine of the scapula forms a $Y$ shape (Fig. 3A, dotted line b), among the
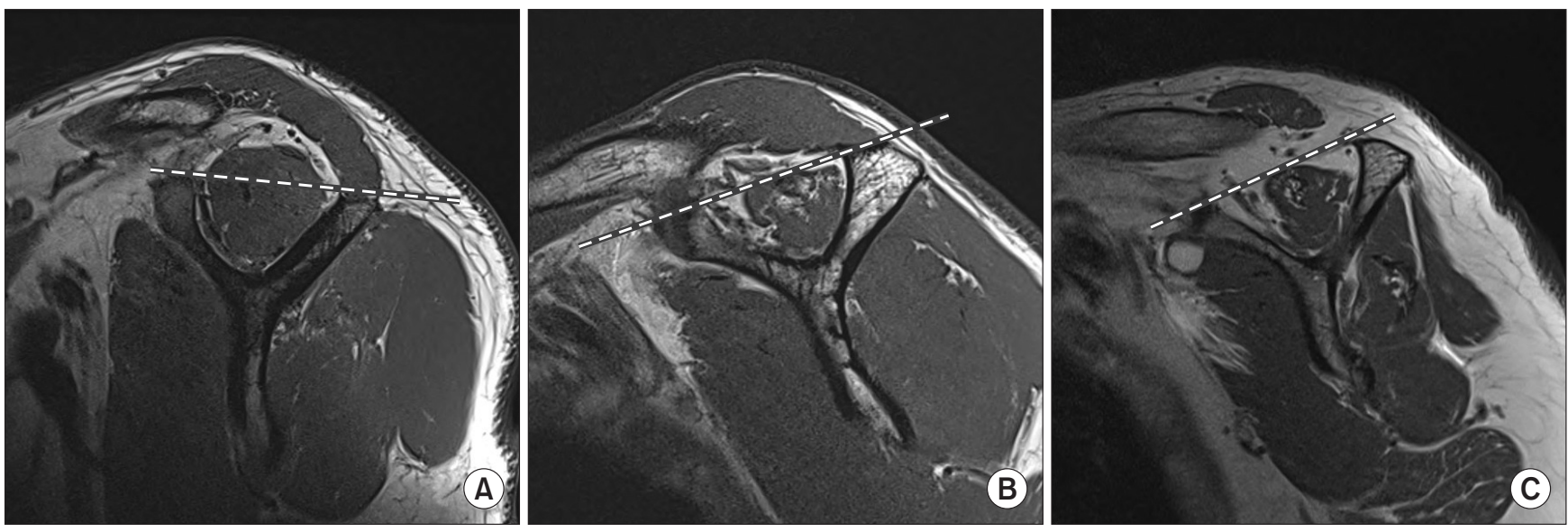

Fig. 2. Preoperative magnetic resonance imaging (MRI) of rotator cuff tear. (A) Preoperative MRI shows atrophy of supraspinatus muscle; grade I by the modified tangent (indicated by a dashed line) sign. (B) Preoperative MRI shows atrophy of supraspinatus muscle; grade II by the modified tangent (indicated by a dashed line) sign. (C) Preoperative MRI shows atrophy of supraspinatus muscle; grade III by the modified tangent (indicated by a dashed line) sign.
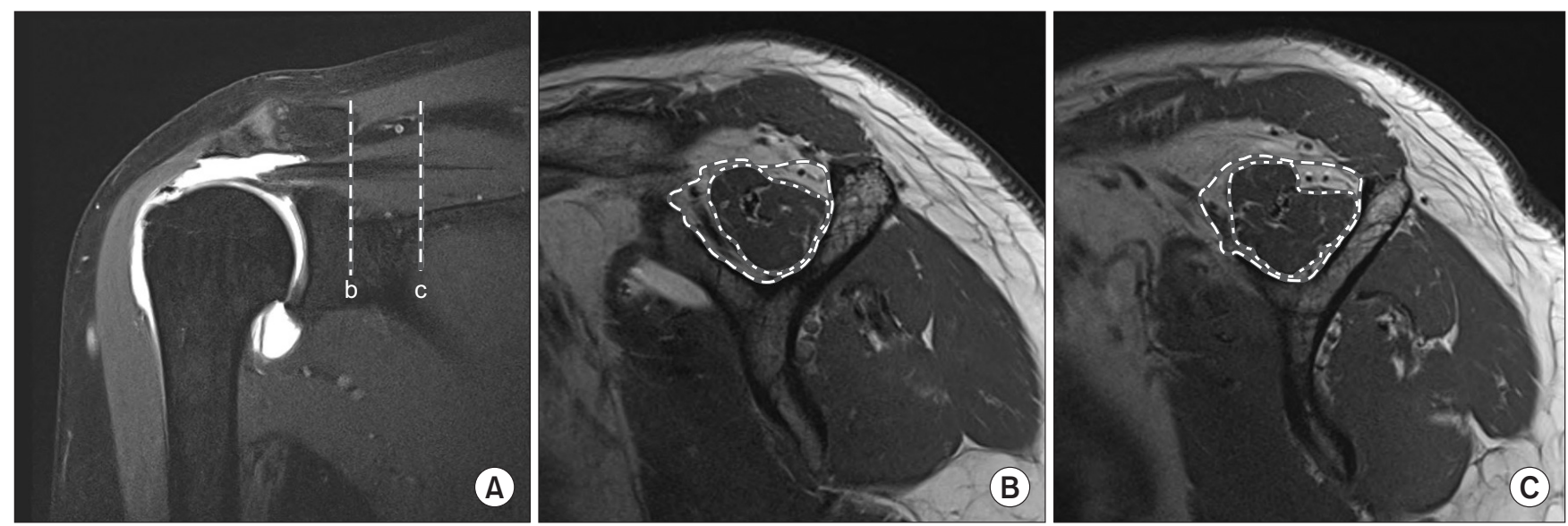

Fig. 3. Measurements of pre- and postoperative magnetic resonance imagings (MRIs). (A) Coronal section of preoperative patient's shoulder MRI. (B) Sagittal section of supraspinatus muscle, Y-shaped view cut from line b in panel A. Outer dash-lined area includes muscle and fat tissue. Inner dot-line is margin of muscle tissue. (C) Sagittal section of supraspinatus muscle, Y-shaped view cut from line c in panel A, at the location of maximum diameter of supraspinatus muscle. Outer dash-lined area includes muscle and fat tissue. Inner dot-line is margin of muscle tissue. 
oblique sagittal planes reported by Thomazeau et al. ${ }^{5)}$ and the OR of the supraspinatus muscle in the oblique sagittal plane was also measured at the maximum diameter of the supraspinatus muscle in the coronal plane (Fig. 3A, dotted line c) using the area measurement of the picture archiving and communication system (PACS, Marosis M-view 5.4; Marotech, Seoul, Korea) (Fig. 3). In addition, the OR of the supraspinatus muscle was also measured in the Y-shaped view of the oblique sagittal plane on immediate postoperative MRI. The OR of the supraspinatus muscle was measured in the same Y-shaped view before and after surgery. One orthopedic surgeon and one orthopedic surgery resident performed the measurements respectively and the averages of the results were used for analysis. Statistical analysis was performed to determine the statistical significance for variables such as the time from the onset of symptoms to surgery, the size of rotator cuff tear, the OR before and after surgery, assessment results according to the Goutallier classification before and after surgery, assessment results of the modified tangent sign before and after surgery, and the OR difference between maximum diameter and Y-shaped view (ODMY) before surgery.

\section{Statistical Analysis}

Statistical analysis was conducted using the SPSS ver. 17.0 (SPSS Inc., Chicago, IL, USA). Inter-observer reliability analysis was performed, and intraclass correlation coefficient (ICC) analysis was also performed (the ICC value of over 0.8 indicates an excellent level of reliability, 0.6-0.8, a good level, 0.4-0.6, a fair level, and 0.4 or less, a poor level, respectively). The Kolmogorov-Smirnov test was used for the test for normality of the measured variables, and paired t-test was performed for preand postoperative assessment results according to the Goutallier classification and modified tangent sign. The Pearson correlation coefficient was used to analyze the relationship between the ODMY and variables, such as the time from symptom onset to surgery, the size of rotator cuff tear, the preoperative OR, the Goutallier classification before and after surgery, and the modified tangent sign before and after surgery. The level of statistical significance was set at $p<0.05$.

\section{Results}

Before surgery, fatty degeneration of the supraspinatus muscle was classified as grade I in 2 patients, grade II in 18 patients, and grade III in 7 patients according to the Goutallier classification. Postoperatively, fatty degeneration was assessed as grade I in 21 patients, grade II in 5 patients, and grade III in 1 patient. As for the modified tangent sign, there were 7 cases of grade I, 17 cases of grade II, and 3 case of grade III before surgery, while there were 21 cases of grade I, 6 cases of grade II and, and 0 case of grade III immediately after surgery. The size of rotator cuff tear was $25.52 \pm 8.22 \mathrm{~mm}(15-44 \mathrm{~mm})$. The cases of supraspinatus tears included 5 patients with a partial tear and 22 patients with a full-thickness tear. Regarding the tears of the infraspinatus muscle, 5 patients had no tear, 3 patients had a partial tear, and 19 patients had a full-thickness tear. The preoperative OR in the Y-shaped view was 52.28 \pm 8.57 (32.5-65.3). In addition, the preoperative OR measured at the maximum

Table 2. Result of Measurements

\begin{tabular}{lcc}
\hline \multicolumn{1}{c}{ Variable } & Value & $p$-value \\
\hline Tear size $(\mathrm{mm})$ & $25.52 \pm 8.22(15-44)$ & $0.558^{\dagger}$ \\
Supraspinatus muscle tear & 5 & \\
Partial-thickness tear & 22 & \\
Full-thickness tear & &
\end{tabular}

Infraspinatus tear

\begin{tabular}{|lc|}
\hline None & 5 \\
\hline Partial-thickness tear & 3 \\
\hline Full-thickness tear & 19 \\
\hline
\end{tabular}

Preoperation

$\begin{array}{lrl}\text { Goutallier classification } & 0.001^{* *} \\ \text { I } & 2 \\ \text { II } & 18\end{array}$

III 7

Modified tangent sign $\quad 0.003^{* *}$

I 7

II 17

III 3

Occupation ratio $\quad 52.28 \pm 8.57(32.5-65.3)$

Maximum diameter occupation $\quad 66.04 \pm 5.53(55.2-74.5)$ ratio

Postoperation

Goutallier classification $0.001^{\star *}$

I 21

II 5

III

Modified tangent sign $\quad 0.004^{\star^{*}}$

I 21

II 6

III 0

Occupation ratio $\quad 63.77 \pm 9.35(37.3-76.1)$

ODMY $\quad 13.76 \pm 10.51(2.38-42.04)$

Values are presented as mean \pm standard deviation (range) or number only. ODMY: occupation ratio difference between maximum diameter and Yshaped view

*Statistically significant.

${ }^{\dagger}$ Kolmogorov-Smirnov test.

${ }^{*}$ Paired t-test. 
diameter location of the supraspinatus muscle was $66.04 \pm 5.53$ (55.2-74.5). The immediate postoperative OR in the Y-shaped view was $63.77 \pm 9.35$ (37.3-76.1). The ODMY was $13.76 \pm$ 10.51 (2.38-42.04) (Table 2). As for the preoperative inter-rater correlation coefficient, it was 0.85 for preoperative OR in the $\mathrm{Y}$ shaped view, 0.81 for OR at the maximum diameter location of the supraspinatus muscle, and 0.89 for immediate postoperative OR. There was a statistically significant correlation between ODMY and postoperative OR in the Y-shaped view and the Pearson correlation coefficient was $0.604(p=0.001)$. In addition, there was also a statistically significant correlation between preoperative OR and ODMY, and the Pearson correlation coefficient was $-0.809(p=0.001)$. Pre- and postoperative Goutallier classifications were statistically significantly correlated with ODMY, and the Pearson's correlation coefficient was -0.579

Table 3. Pearson Correlation Coefficient ( $p$-Value)

\begin{tabular}{ll}
\hline \multicolumn{1}{c}{ Variable } & \multicolumn{1}{c}{ ODMY } \\
\hline Onset to operation & $-0.199(0.319)$ \\
\hline Tear size & $0.136(0.497)$ \\
\hline Preoperation occupation ratio & $-0.809(0.001)^{\star}$ \\
\hline Preoperation Goutallier classification & $-0.579(0.002)^{\star}$ \\
\hline Preoperation modified tangent sign & $0.279(0.158)$ \\
\hline Postoperation Goutallier classification & $-0.386(0.047)^{\star}$ \\
\hline Postoperation modified tangent sign & $0.146(0.486)$ \\
\hline Postoperation occupation ratio & $0.604(0.001)^{\star}$ \\
\hline
\end{tabular}

Values are presented as Pearson correlation coefficient of pre- and postoperative Goutallier classification ( $p$-value).

ODMY: occupation ratio difference between maximum diameter and Yshaped view.

${ }^{*}$ Statistically significant. $(p=0.002)$ and $-0.386(p=0.047)$, respectively (Table 3$).$

\section{Discussion}

The atrophy and fatty degeneration of the rotator cuff are reported to be closely related to the prognosis of rotator cuff repair. ${ }^{16,17)}$ Taking a conventional approach to assess fatty degeneration, Goutallier attempted to measure the degree of fatty degeneration of the supraspinatus muscle in the Y-shaped view where the scapular body and spine of the scapula form a $Y$ shape among the oblique sagittal planes. ${ }^{3}{ }^{3}$ However, the comparison between pre- and postoperative MRI scans performed in our institution showed that the degree of fatty degeneration was significantly different among the patients (Fig. 4, 5). A number of studies for the accurate assessment of fatty degeneration of the retracted supraspinatus muscle have been conducted. Fukuta et al. ${ }^{16)}$ pointed out that it is difficult to assess the degree of fatty degeneration of the supraspinatus muscle using the OR of the supraspinatus muscle on a sagittal view. Thus, they assessed the OR in the first, second and third inner planes of the supraspinatus muscle in the Y-shaped view of the MRIs divided into 5-mm segments, and analyzed the statistical significance. As a result, they recommended assessing OR on the second inner plane in the Y-shaped view. In this study, the shape of the supraspinatus muscle was examined in the coronal plane rather than examining a specific inner plane image, and OR was measured by setting the maximum diameter location of the retracted supraspinatus muscle as the measurement position. Then, we analyzed the statistical significance for the relationship between the difference between ORs measured at the maximum diameter and in the Y-shaped view (ODMY) and the postoperative OR. The reason why OR was measured at the maximum diameter location of the retracted supraspinatus muscle is that this meth-
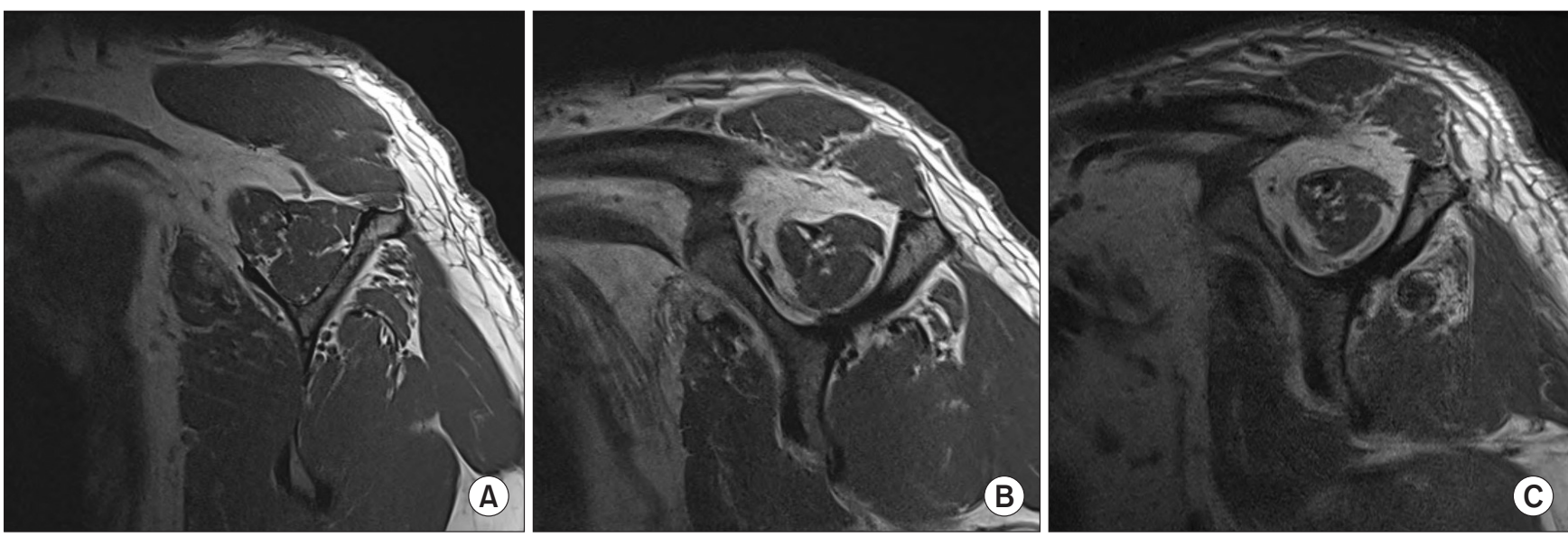

Fig. 4. Magnetic resonance imaging (MRI) of a 61-year-old male patient with rotator cuff tear. (A) Sagittal view of preoperative T1-weighted MRI at location of maximum diameter of supraspinatus muscle measured from coronal section of shoulder MRI. (B) Sagittal view of preoperative T1-weighted MRI of supraspinatus muscle, Y-shaped view in coronal section of shoulder MRI. (C) Sagittal view of postoperative T1-weighted MRI of supraspinatus muscle, Y-shaped view in coronal section of shoulder MRI. 

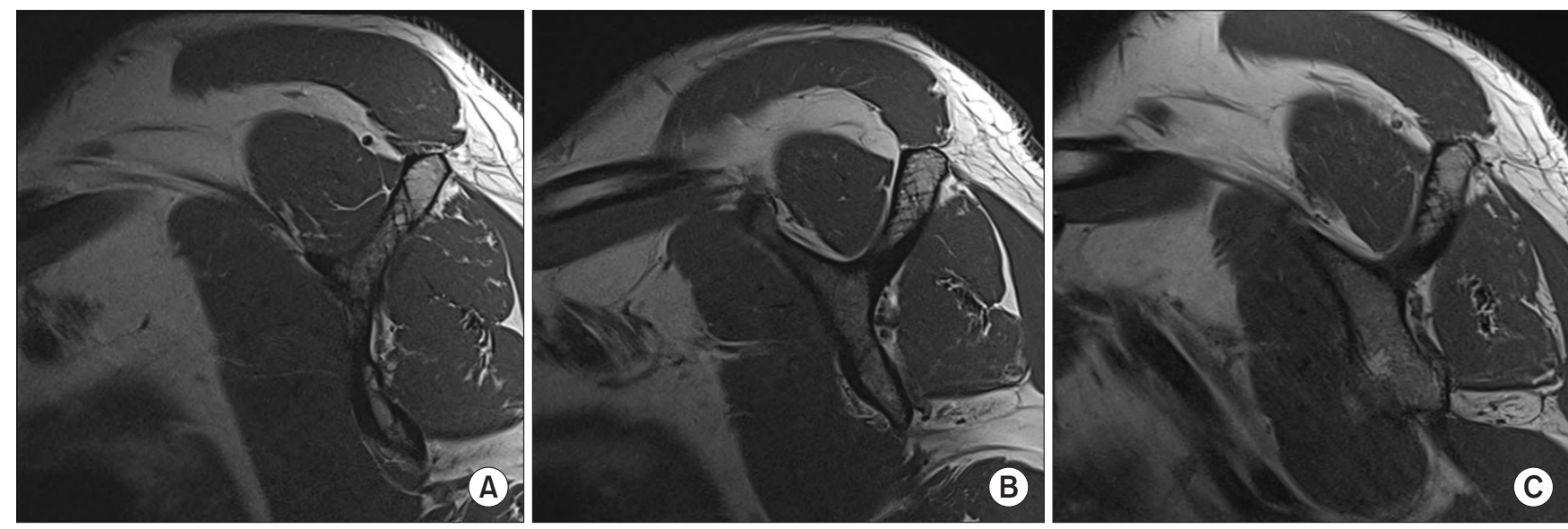

Fig. 5. Magnetic resonance imaging (MRI) of a 51-year-old male patient with rotator cuff tear. (A) Sagittal view of preoperative T1-weighted MRI at location of maximum diameter of supraspinatus muscle measured from coronal section of shoulder MRI. (B) Sagittal view of preoperative T1-weighted MRI of supraspinatus muscle, Y-shaped view in coronal section of shoulder MRI. (C) Sagittal view of postoperative T1-weighted MRI of supraspinatus muscle, Y-shaped view in coronal section of shoulder MRI.

od allows accurate assessment since the position is the location where the cross section of the muscle is largest and there is also the advantage that assessment is less affected by MRI performed than when the measurement position was set as in Fukuta et al. ${ }^{16)}$ As ODMY became larger, the postoperative OR in the $\mathrm{Y}$ shaped view was increased, which indicates that the degree of fatty degeneration of the supraspinatus muscle is small. A small ODMY measured in the Y-shaped view can be considered to indicate that fatty degeneration of the supraspinatus muscle occurred in the patient. In the patients showing a large ODMY, the degree of fatty degeneration measured in the Y-shaped view was high, but this is likely to be a result of making the measurement at the myotendinal junction due to muscle atrophy and the degree of fatty degeneration of the supraspinatus muscle may be actually low. Many studies have reported that fatty degeneration and atrophy of muscles are important factors in the prognosis and treatment outcomes of patients. ${ }^{16,18,19)}$ In addition, muscles showing atrophy and fatty degeneration are known to show reduced muscle elasticity and be less suitable for recovery. Thus, we concluded that it is important to examine the state of the supraspinatus muscle itself as well as fatty degeneration of the rotator cuff before surgery to establish the treatment plan of each patient, such as arthroplasty, open repair, and tendon transfer. In our institution, we perform MRI of T1-weighted images in the oblique sagittal plane up to the location maximally proximal to MRI measurement coils to accurately assess the degree of fatty degeneration of the supraspinatus muscle in the MRI test of patients suspected to have a rotator cuff tear. By this method, we try to avoid making selection errors in patients with the rotator cuff tear involving the retracted supraspinatus muscle, especially not to erroneously perform overtreatment such as arthroplasty in patients who can be treated only by a rotator cuff repair. This study has some limitations. First, this study is a retrospective study and not a randomized trial. In addition, since the patients who did not undergo pre- and postoperative MRIs in our institution for personal reasons could not be included in the study, a small number of patients were included in this study. In addition, different interpretations from the results of this study can be made if the severity of fatty degeneration is not similar degeneration at the maximum diameter location of the supraspinatus muscle and in the Y-shaped view. However, the significance of this study can be found in the fact that this study is one of the recent studies on accurate assessment of fatty degeneration in patients with rotator cuff tear and has presented an analysis by one of accurate evaluation methods.

\section{Conclusion}

The evaluation of fatty degeneration of the retracted supraspinatus muscle should be conducted not only in the Y-shaped view but also at the location of the maximal diameter of the supraspinatus muscle not to make a therapeutic that is not suitable for the patient's condition.

\section{References}

1. lannotti JP. Full-thickness rotator cuff tears: factors affecting surgical outcome. J Am Acad Orthop Surg. 1994;2(2):87-95.

2. Hamano N, Yamamoto A, Shitara H, et al. Does successful rotator cuff repair improve muscle atrophy and fatty infiltration of the rotator cuff? A retrospective magnetic resonance imaging study performed shortly after surgery as a reference. J Shoulder Elbow Surg. 2017;26(6):967-74.

3. Goutallier D, Postel JM, Bernageau J, Lavau L, Voisin MC. Fatty muscle degeneration in cuff ruptures. Pre- and postoperative evaluation by CT scan. Clin Orthop Relat Res. 1994;(304):78- 
83.

4. Gladstone JN, Bishop JY, Lo IK, Flatow EL. Fatty infiltration and atrophy of the rotator cuff do not improve after rotator cuff repair and correlate with poor functional outcome. Am J Sports Med. 2007;35(5):719-28.

5. Thomazeau H, Rolland Y, Lucas C, Duval JM, Langlais F. Atrophy of the supraspinatus belly. Assessment by MRI in 55 patients with rotator cuff pathology. Acta Orthop Scand. 1996; 67(3):264-8.

6. Liem D, Lichtenberg S, Magosch P, Habermeyer P. Magnetic resonance imaging of arthroscopic supraspinatus tendon repair. J Bone Joint Surg Am. 2007;89(8):1770-6.

7. Shen PH, Lien SB, Shen HC, Lee CH, Wu SS, Lin LC. Longterm functional outcomes after repair of rotator cuff tears correlated with atrophy of the supraspinatus muscles on magnetic resonance images. J Shoulder Elbow Surg. 2008;17(1 Suppl): 1S-7S.

8. Jo $\mathrm{CH}$, Shin JS. Changes in appearance of fatty infiltration and muscle atrophy of rotator cuff muscles on magnetic resonance imaging after rotator cuff repair: establishing new time-zero traits. Arthroscopy. 2013;29(3):449-58.

9. Vidt ME, Santago AC 2nd, Tuohy CJ, et al. Assessments of fatty infiltration and muscle atrophy from a single magnetic resonance image slice are not predictive of 3-dimensional measurements. Arthroscopy. 2016;32(1):128-39.

10. Gerber C, Schneeberger AG, Hoppeler H, Meyer DC. Correlation of atrophy and fatty infiltration on strength and integrity of rotator cuff repairs: a study in thirteen patients. J Shoulder Elbow Surg. 2007;16(6):691-6.

11. Yamaguchi $H$, Suenaga $N$, Oizumi N, Hosokawa Y, Kanaya F. Will preoperative atrophy and fatty degeneration of the shoul- der muscles improve after rotator cuff repair in patients with massive rotator cuff tears? Adv Orthop. 2012;2012:195876.

12. Chung SW, Kim SH, Tae SK, Yoon JP, Choi JA, Oh JH. Is the supraspinatus muscle atrophy truly irreversible after surgical repair of rotator cuff tears? Clin Orthop Surg. 2013;5(1):55-65.

13. Deniz G, Kose O, Tugay A, Guler F, Turan A. Fatty degeneration and atrophy of the rotator cuff muscles after arthroscopic repair: does it improve, halt or deteriorate? Arch Orthop Trauma Surg. 2014;134(7):985-90.

14. Gerber C, Meyer DC, Schneeberger AG, Hoppeler H, von Rechenberg B. Effect of tendon release and delayed repair on the structure of the muscles of the rotator cuff: an experimental study in sheep. J Bone Joint Surg Am. 2004;86(9):1973-82.

15. Fuchs B, Weishaupt D, Zanetti M, Hodler J, Gerber C. Fatty degeneration of the muscles of the rotator cuff: assessment by computed tomography versus magnetic resonance imaging. J Shoulder Elbow Surg. 1999;8(6):599-605.

16. Fukuta S, Tsutsui T, Amari R, Wada K, Sairyo K. Tendon retraction with rotator cuff tear causes a decrease in cross-sectional area of the supraspinatus muscle on magnetic resonance imaging. J Shoulder Elbow Surg. 2016;25(7):1069-75.

17. Zanetti M, Gerber C, Hodler J. Quantitative assessment of the muscles of the rotator cuff with magnetic resonance imaging. Invest Radiol. 1998;33(3):163-70.

18. Kuzel BR, Grindel S, Papandrea R, Ziegler D. Fatty infiltration and rotator cuff atrophy. J Am Acad Orthop Surg. 2013; 21(10):613-23.

19. Warner JJ, Higgins L, Parsons IM 4th, Dowdy P. Diagnosis and treatment of anterosuperior rotator cuff tears. J Shoulder Elbow Surg. $2001 ; 10(1): 37-46$. 